\title{
3D Laser Imaging Reconstruction of Scenes Using New Photometric Gaussian Beam Range Equation
}

\author{
Mounir Boujenab ${ }^{*}$ and Noureddine Eddeqaqi Cherkaoui \\ Laboratory of Atomic, Mechanics, Photonics and Energy, Department of Physics, Faculty of Science, University Moulay Ismail \\ Meknes 50000, Morocco
}

\begin{abstract}
In this article the problem of three-dimensional (3-D) reconstruction of scenes from terrestrial laser rangefinder data is processed, we present the simulations results of radiometric range function of the laser range finder imaging system for better optimization of maximum range detection with a given SNR (Signal-to-noise ratio). The study proposes a new reformulation of the radiometric Gaussian beam range equation on the basis of the photodetector active area. The adjustment of the optical and geometrical characteristics of the laser range finder so defined can be used to minimize the signal span to avoid the saturation of the detector cell and improve the ranging measurements (accuracy and range) for a 3D imaging application.
\end{abstract}

Key words: Laser ranging and imaging, signal to noise ratio, Gaussian beam, photometric range equation.

\section{Nomenclature}

$P_{e} \quad$ Emitted laser power

$P_{r} \quad$ Received power

$R \quad$ Measuring distance (range)

$S N R$ Signal to nose ratio

$N E P$ Nose equivalent power

$C \quad$ Light velocity

$V \quad$ Atmospheric visibility

$f \quad$ Reception lens focal

$d$ Distance between the photodetector and the focal plane

$D \quad$ Objective lens diameter

$D_{\omega_{i}}$ Image waist distance from emission optic

$R_{p} \quad$ Reception pupil radius

$d_{d}$ Gaussian beam diameter at the output of the collimation

$d_{d} \quad$ optic

$A_{t} \quad$ Target area

$r_{t} \quad$ Target radius

\section{Greek letters}

\section{$\Delta t \quad$ Time of flight}

$\theta_{r} \quad$ Half angle of laser beam convergence

$\theta_{e} \quad$ Half angle of laser beam divergence

$\Delta_{\varphi} \quad$ Angle convergence between the emission axis and the

$\Delta_{\varphi} \quad$ reception axis

$\tau_{e} \quad$ Transmitter optical efficiency

$\tau_{r} \quad$ Receiver optical efficiency

*Corresponding author: Mounir Boujenab, Ph.D. student, research fields: optics, image and signal processing. E-mail: mounirboujenab@gmail.com.

\author{
$\tau_{\text {atm }} \quad$ Atmosphere transmission factor \\ $\omega_{i} \quad$ Image waist \\ $\rho \quad$ Target reflectance (Albedo) \\ $\delta \quad$ Parllaxe of the rangefinder optical system.
}

\section{Introduction}

The technique of mobile laser imaging or LDAR; (Light detection and ranging), allows obtaining a 3-D points cloud, by scanning of the scene in order to generate 3-D photorealistic urban models [1]. The quality and density of information obtained (points cloud formation), are a considerable advantage in terms of measurement accuracy. The laser imaging system is based on its active detection module on a laser range finder for measuring distances of the targets with a high spatial resolution $[2,3]$. There are several telemetry methods for estimating distances, but they can be grouped into two broad categories: coherent or indirect methods and direct methods. The most used laser imaging technique is the direct method, called TF (Time of flight), this technique is based on the transmission of a short pulse of electromagnetic radiation and receiving the back return signals diffused from a target. The time between the transmission and reception of the pulse or time of flight $\Delta t$ is measured 
and the distance $R$ is calculated on the basis of the relation $R=c . \Delta t / 2$.

On the laser telemetry, the mathematical expression of the relationship between the detected power (the transmitted power is known) and the measured distance is known as radiometric range equation. Since the light pulse is reflected by the target, then this equation depends on the characteristics of the light pulse, target and atmospheric attenuation parameters.

In this paper we will first evaluate the effect of Gaussian profile introduced into the radiometric range function [4] in the estimation of the maximum distance between the laser imaging system and the scene to reproduce, for a given SNR (Signal-to-noise ratio), for a better measurement accuracy for all values of range distances regardless of the two cases; total reflection of the laser beam or partial reflection [5]. Second, starting from the study of the returned signal span effect on the receiving cell [6] we propose a new Gaussian reformulation of radiometric range equation based on geometric and photometric calculation of the illuminated effective area. We show that for an imaging application, the distance between the imaging laser and the scene should be above a saturation threshold of the receiver module.

\section{The Radiometric Range Equations}

The radiometric range equation measures the returned power radiation reflected by a target. This is the technique that will allow us to define the spatial sensor performance and especially know the maximum distance where the laser system can be situated for always having a correct image, from the fourth following parameters:

(1) The power density received by the target,

(2) The attenuation factors and atmospheric extinction of electromagnetic radiation along the path between the laser transmitter and the optical receiving system,

(3) The power received at the input of the receiving optical system,
(4) The power received by the photoreceptor.

Then we can establish the radiometric range equation of the system for normal incidence and totally reflected beam. In this case the target dimensions are greater than the laser spot, i.e. $A_{t} \geq\left(\theta R+d_{d}\right)$. The radiometric range equation is given hereafter [4].

$$
P_{r}=\frac{\rho \tau_{a t m}^{2} \tau_{e} \tau_{r} D^{2}}{8 R^{2}} P_{e}
$$

where, as in the case of normal incidence and partially reflected beam, the target dimensions are smaller than the laser spot, i.e. $A_{t} \geq\left(\theta R+d_{d}\right)$,

$$
P_{r}=\frac{\rho \tau_{a t m}^{2} \tau_{e} \tau_{r} D^{2} A_{t}}{8 \pi \theta^{2} R^{4}} P_{e}
$$

which is noted that in the general case of an impact with an angle $\varphi$ on the target plane the previous expressions must be multiplied with $\cos \varphi$ [7].

\section{Signal to Noise Ratio (SNR)}

The SNR measures the quality of the transmission of information in relation with different noises (thermal noise, electronic amplifier noise, quantum shot noise, dark current noise, background noise, etc.) and determines the accuracy of the distance measurement [8-10]. It is defined to be the ratio of the received power to the noise.

$$
S N R=\frac{P_{r}}{N E P}
$$

where, $N E P$, the noise equivalent power, is the power (in watts) received by the detector corresponding to the noise.

\subsection{The Maximum Distance between the Laser and the Target According to the Visibility}

The purpose of this calculation is to find the maximum range of the laser rangefinder i.e. the maximum distance between the target and the laser source function of the visibility $V$ [11] in order to perform the detection with an optimal precision 
accuracy above which the quality of the reproduced image is affected.

For this, we search the maximum distance $R_{\max }$ that minimizes the difference between a fixed $S N R$ and the ratio of the received power, function of the visibility and this distance, and the $N E P$ system.

We calculate, in the case of a totally reflective target:

$$
\min _{R \in I R} S N R-\left(P_{e} \rho \tau_{e} \tau_{r} \frac{D^{2}}{8}\right) \frac{\exp ^{-2 \gamma(V) R}}{R^{2}}(4)
$$

and for a partially reflective target:

$$
\min _{R \in I R} S N R-\left(P_{e} \rho \tau_{e} \tau_{r} \frac{D^{2} A_{t}}{8 \pi \theta^{2}}\right) \frac{\exp ^{-2 \gamma(V) R}}{R^{4}}(5)
$$

where, $A_{t}$ is the target area. We set $S N R=10$, and we assume, for this case, that the emitted power is uniformly distributed and not Gaussian.

\subsection{Numerical Simulation}

The numerical simulation presented in Fig. 1 is performed by the technical characteristics of the eye safety Er : Glass laser range finder MK81 from Kigre Inc. [4], the $N E P$ of the system should be about $30 \mathrm{nW}$ according to [12], $P_{e}=0.33 M W, \rho=0.21, D=$ $45 \mathrm{~mm}, \tau_{e}=0.85, \tau_{r}=0.45, A_{t}=2.3 \times 2.3 \mathrm{~m}^{2}$ and $\theta=0.6 \mathrm{mrad}$.

\subsection{Discussion of Results}

For the SNR fixed at 10 and a maximum visibility of $20 \mathrm{~km}$, the maximum detection distance for total reflection is $3,529 \mathrm{~m}$, and it is $2,842 \mathrm{~m}$ for partially reflected beam. In the case of total reflection, the distance $R_{\max }$ is very important, especially as the visibility is better, but it means we always have a target area greater than or equal to the laser spot (the area of the target is not an included parameter in the radiometric rang equation). Therefore, in the case of a laser imaging process, it is required to appreciate the targets dimensions in advance to ensure the correct position of the system and have a clear image.

In the case of partial reflection, the maximum distance $R_{\max }$ is lower, it is a normal result, since a large portion of the laser radiation (the number of detected photon) is not captured in return by the detector, decreasing the received power and minimizing the range. Also, in the case of partial reflection, it is required to ensure the dimensions of the target related to the laser spot size.

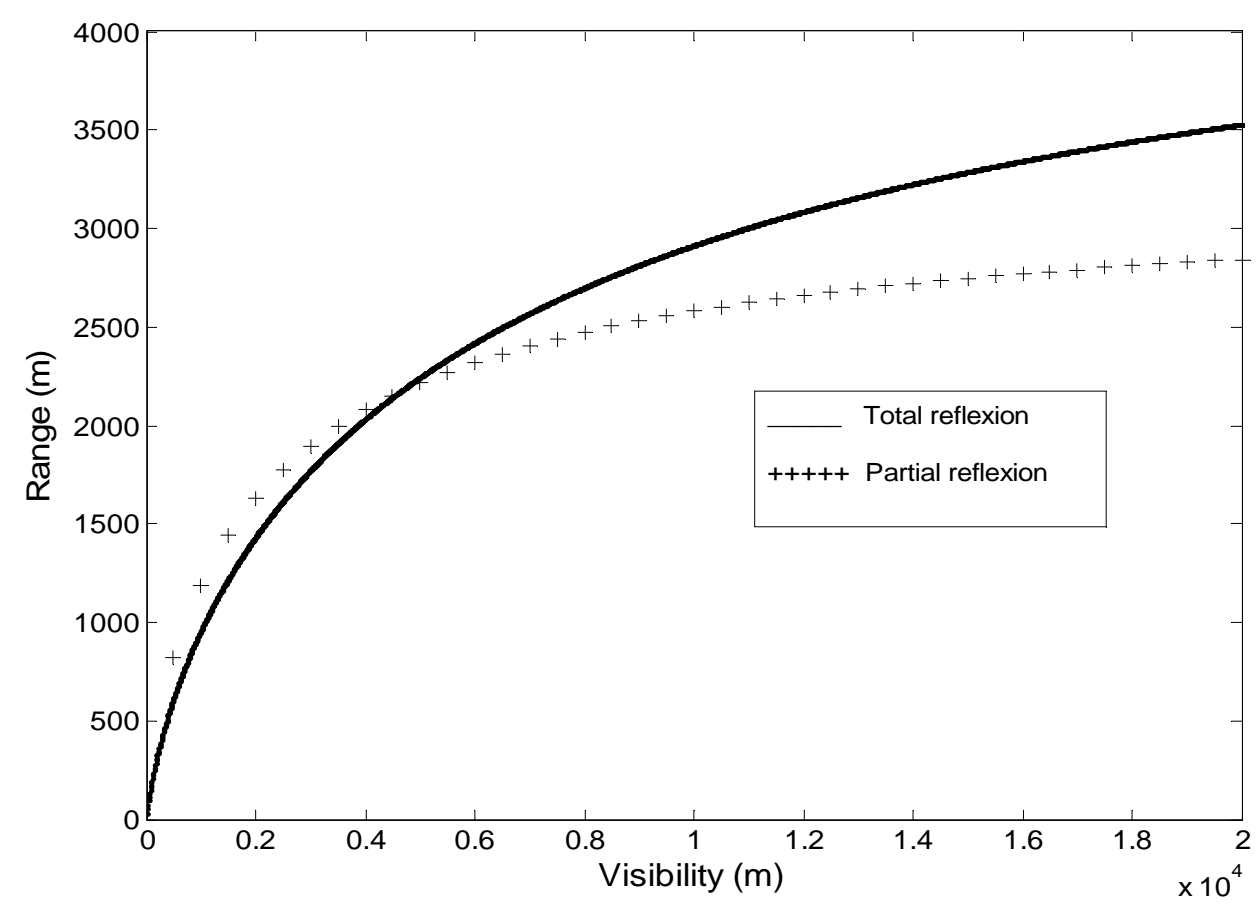

Fig. 1 Maximum range function of visibility for totally and partially reflected beam. 
In summary, for these two cases (total reflection and partial reflection) and for laser imaging application, it is always essential to evaluate correctly the target receiving area in order to work with one of the radiometric rang function that optimizes the distance between the target to reconstruct and the laser-camera device, before introducing it into the image processing algorithms.

\section{Gaussian Range Equation Maximum Distance}

The Gaussian range equation is given by [4]:

$$
P_{r}=\left(\frac{P_{e} \rho \tau_{e} \tau_{r} D^{2}}{8}\right)\left[\frac{\exp ^{-2 \gamma(V) R}}{R^{2}}\right]\left[1-\exp \left(\frac{-2 r_{t}^{2}}{\theta^{2} R^{2}}\right)\right]
$$

We will calculate now as previously:

$$
\min _{R \in I R} S N R-\left(\frac{P_{e} \rho \tau_{e} \tau_{r} D^{2}}{8}\right)\left[\frac{\exp ^{-2 \gamma(V) R}}{R^{2}}\right]\left[1-\exp \left(\frac{-2 r_{t}^{2}}{\theta^{2} R^{2}}\right)\right]
$$

where, $r_{t}$ is the target radius. Keeping the SNR at 10, the result of numerical test is given below

For the numerical computation with a Gaussian distribution profile of emitted power and SNR $=10$, it is noted that the maximum detection distance is 2,866 $\mathrm{m}$ for a visibility of $20 \mathrm{~km}$, it is practically the same distance as for the case of partial reflection since we worked with the same target size. It is also verified that the Gaussian radiometric range function provides maximum detection distance less than that given by the two other radiometric range functions for less than $6 \mathrm{~km}$ of visibility, which is very logical in bad condition of visibility, and allows in fact the optimal use of laser rangefinder without losing signal quality.

Also, the Gaussian radiometric range function minimizes optimally this distance more than it provides totally reflected beam range function which gives much exaggerated detection distances. Furthermore, it is also interesting to note that the Gaussian profile of radiometric range equation provides best maximum range as the SNR of the system decreased. So, we note that the maximum detection distance is $3,429 \mathrm{~m}$ for $20 \mathrm{~km}$ of visibility, which is more important than $3,314 \mathrm{~m}$ provides by the partial reflection range equation for the same visibility. This result is very important if we consider bad conditions of visibility when the returned power is sensibly affected [13, 14].

In practical case of a laser imaging process, the targets that form the scene to capture must be on the

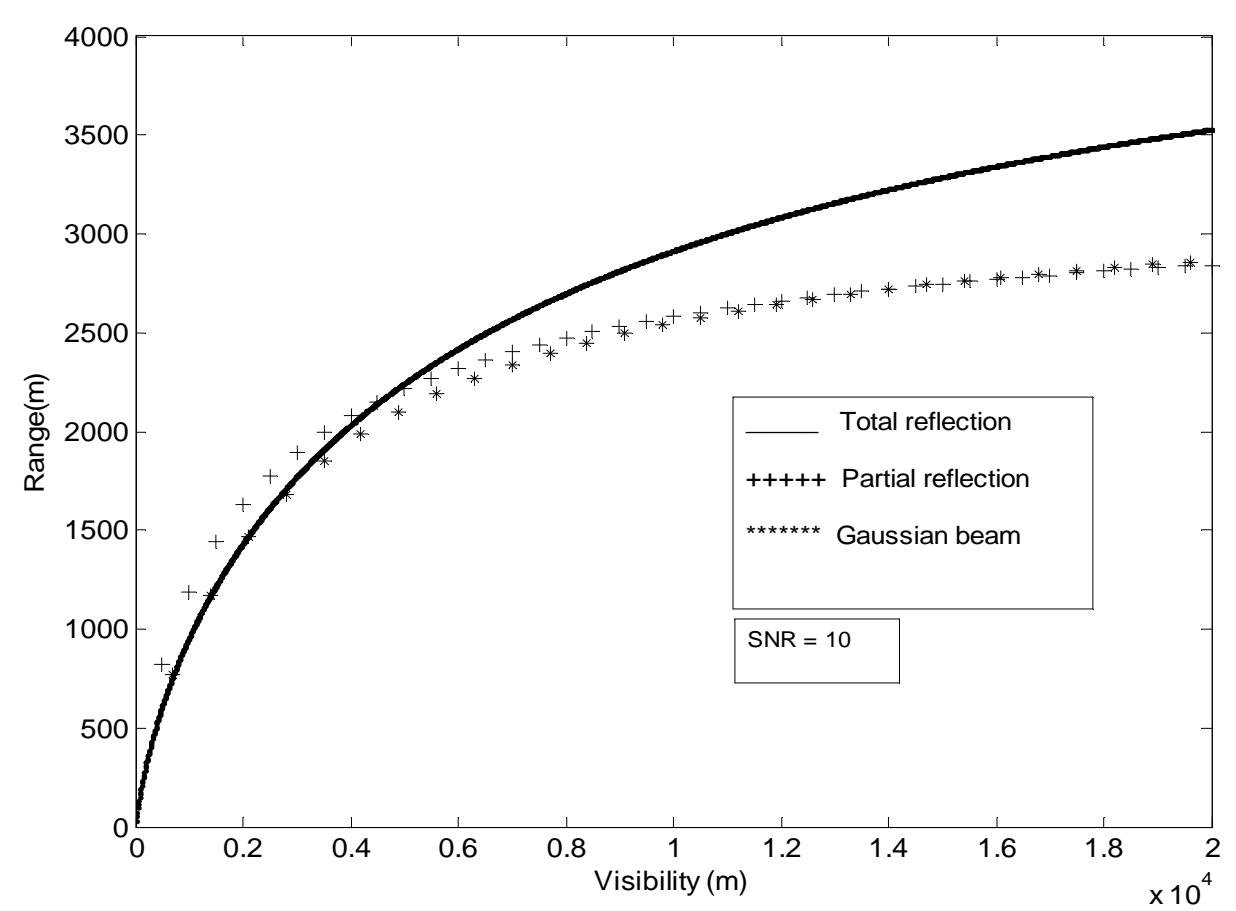

Fig. 2 Maximum range function of visibility comparison for the three expressions (4),(5)and (7),with $S N R=10$. 


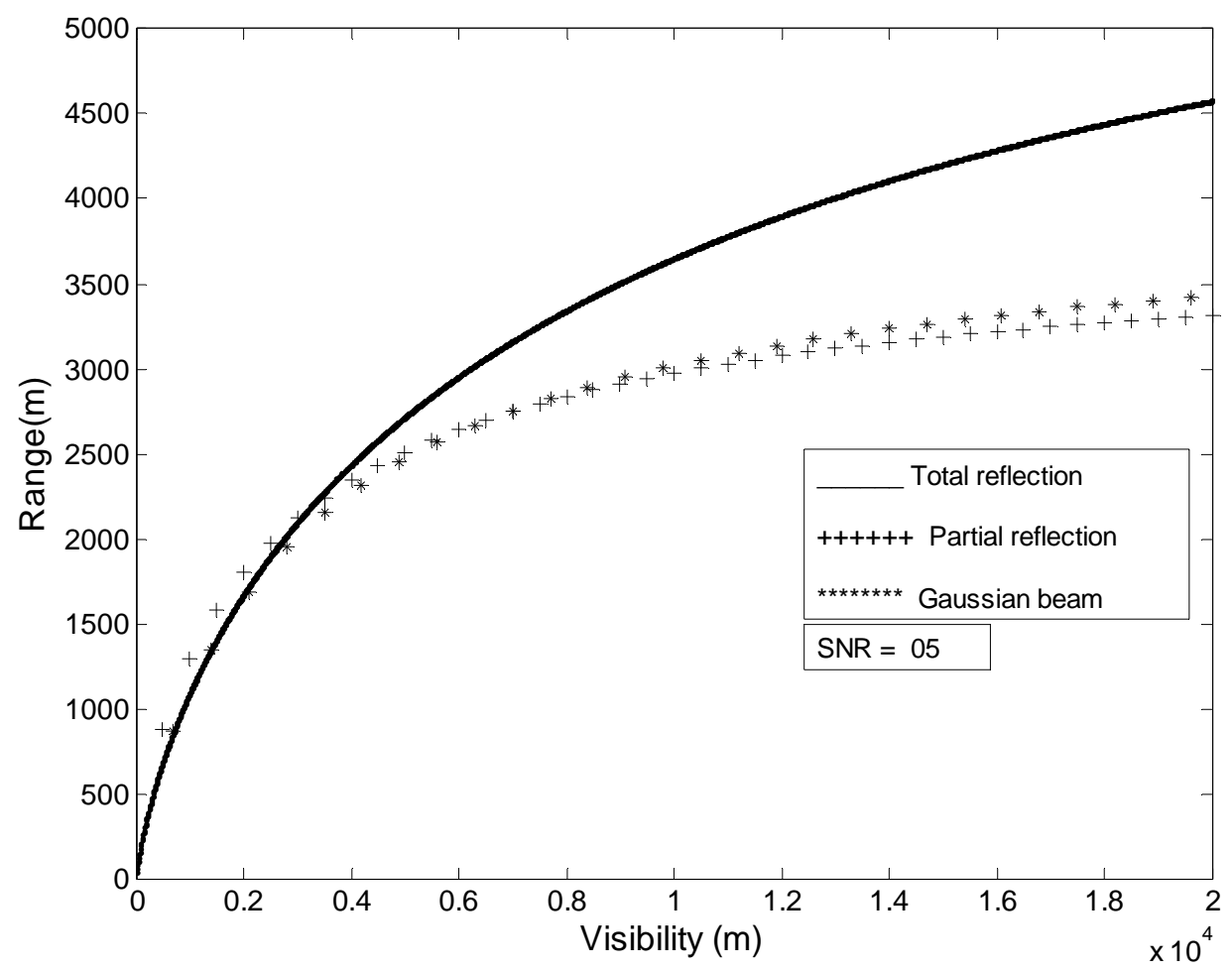

Fig. 3 Maximum range function of visibility comparison, for the three expressions (4), (5) and (7), with SNR = 5.

optical axis of the laser system to ensure a normal incidence (Lambertian cosine $=1$ ) and increase the energy reflected in the sensor direction, but in reality these targets have practically topographical structure (case of a mobile mapping application or a 3D scene restitution) so that a near target can be totally reflective, while another target more distant on the same optical axis can be partially reflective for the same image acquisition. Also in the case of a dynamic detection (the laser imaging device is embedded on a mobile machine; car, robot, etc. [15-18]) the distance variation between the targets and the imaging device can cause the change of the nature of these targets (totally reflective or partially reflective) which will affected the range accuracy. So, a radiometric range function that can be justified for one case can't be so in the other and vice versa, since there will be an over- or underestimation of the detection distance which will affect the quality of the image.

As against, the use of a radiometric Gaussian beam range equation will fix this problem since we will not have to deal with the problem of totally or partially reflective targets. Furthermore, only an average estimation of the target radius is requested.

The simulation which follows in Fig. 4 shows the influence of a variation of the target radius on the power detected by the sensor.

First, we note that for optimal detection range (which is around $100 \mathrm{~m}$ for our case) the targets radius variation has practically no influence on the detected power; it means that the quality of produced images is maximum even for a target radius variation from $0.1 \mathrm{~m}$ to $10 \mathrm{~m}$. Furthermore, the detected power is significantly affected for long distances (which are also not recommended for high quality images), especially for very small $r_{t}$, but no change more than $r_{t}$ increases (practically no change for the $r_{t}$ great than $1 \mathrm{~m}$, which represent interesting targets for mobile mapping and others imaging applications). We can deduce that for targets radius greater than $1 \mathrm{~m}$, the reemitted detected power is perfectly stable, so the use of radiometric Gaussian range function is well justified and remains accurate even with variable dimensions targets. 


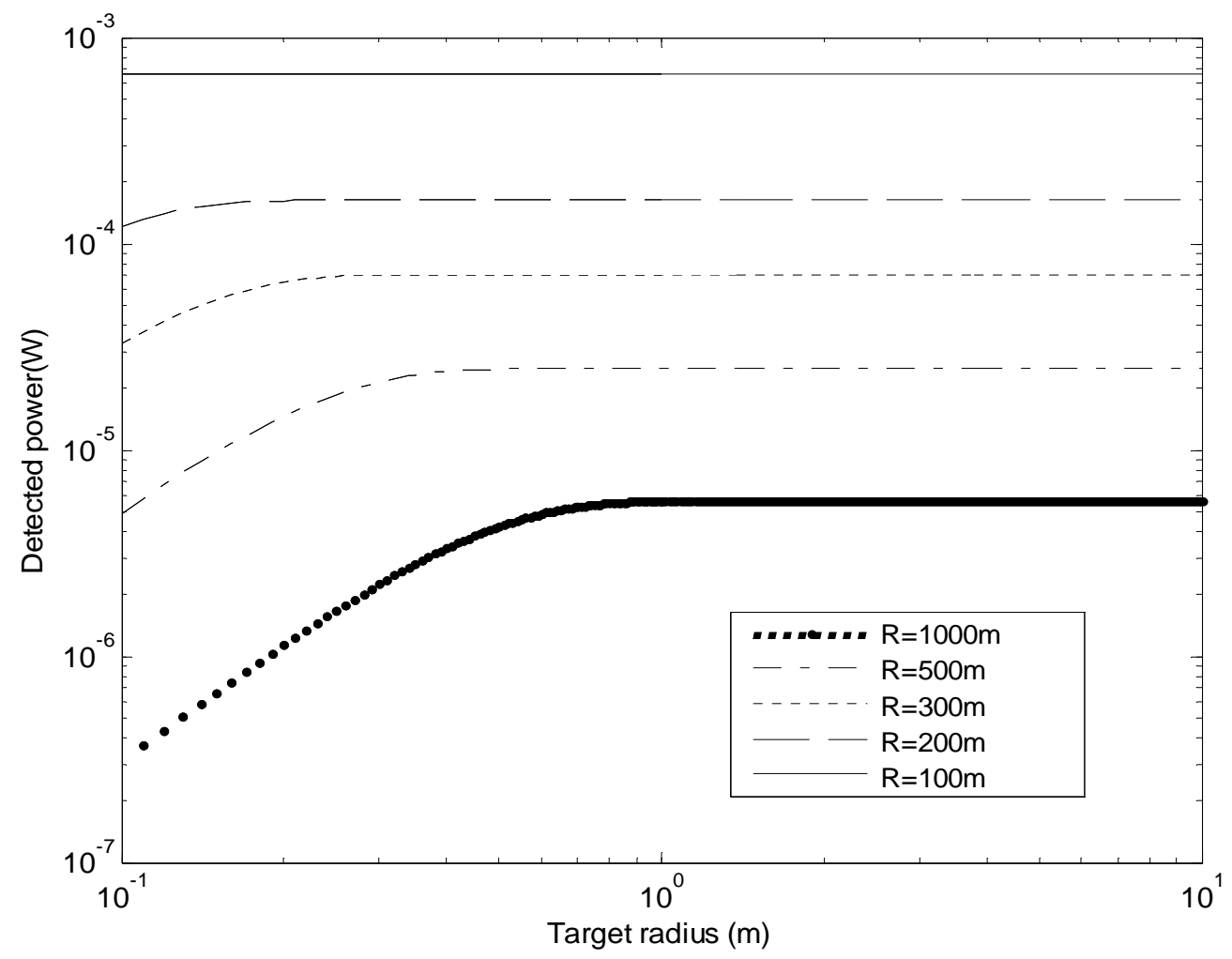

Fig. 4 Effect of the target radius change on the detected power for different measuring ranges.

\subsection{Discussion}

In the case of a dynamic detection which can be characterized by a lateral movement of the laser imaging system relatively to its environment or by a movement along the optical transmission-reception axis, the variation of the detection distance, which is the distance of the image taking, influences the nature of the targets (totally reflective or partially reflective) for the same active sequence of distance calculation and image taking, which leads to erratic measurements and therefore, a degradation of the quality of reconstructed pictures; because it is impossible to change, in practice, simultaneously the radiometric range function that is in accordance with the appropriate range. As against, the introduction of a radiometric range function on the basis of the Gaussian beam function into the algorithms for computing distances and image processing solves completely this problem, and improves significantly the calculation accuracy .

\section{The New Photometric Gaussian Range Equation Reformulation}

The radiometric range equations given by Eqs. (1), (2) and (6) indicate that the received signal by the rangefinder photodetector decreases in proportion to the inverse square of the distance for a totally reflective target and for the Gaussian beam model, and in proportion to the inverse quadruple of the distance for a partially reflective target. These equations give erratic results in a first approximation for short ranges, considering the geometry, the positioning and the characteristics of the optical elements of the system; The optical axis of the transmitting and receiving system are separate, so the image spot from the target illuminated by the laser moves on the detector plane, perpendicular to the optical axis of reception and in the direction of the emission optical axis. In addition, the photodetector is generally placed exactly in the focus of the receiving lens for a measure to "infinity", if the target is near, its image is defocused. The incident 
energy on the surface of the photodetector is thus changed according to the distance depending to these two additional effects. Mastering these effects is useful to reduce the span of the incident signal on the photodetector and avoid saturation of the photodetectors and electronic amplifiers that induce strong errors on the range measurement. A study in the general case using the theory of photometry is already developed. The second method, approximate, is the work subject of the LETI DSYS laboratory [6], provides quick and easy numerical simulation of the optical power received by the photodetector .

Our objective in this section is to demonstrate the validity of the Gaussian model of the photometric range function, and that it gives more accurate results for all range distance, abstraction made for a totally reflected or partially reflected beam. Here, we will calculate the effective reception area of the photodetector before being introduced into the radiometric range equations. The optical power received by the rangefinder is directly proportional to the surface of the receiving optical. We will highlight the changes made by the consideration of the optical and geometrical parameters of the system. The effective area of reception is given by [6, 19]:

$$
A_{e f f}=\frac{\pi R_{p}^{2}}{\rho P_{e}} \int_{0}^{\infty} \int_{0}^{2 \pi} E_{t}\left(r_{t}, R\right) \cdot \Phi\left(r_{t}, R\right) \cdot r_{t} d r_{t} d \varphi_{t}
$$

Note that $d A_{t}=r_{t} d r_{t} d \varphi_{t}$ is the target surface element diffusing a portion of the incident flux entering through the radius of the reception lens $\left(R_{p}\right)$, while $\Phi_{t}\left(r_{t}, R\right)$ is the part the incident flux on the receiving lens that will be accepted by the detector surface, this flux is proportional to the intersection area between the confusion disc on the plane of the detector and the detector surface as shown in Fig. $5, E_{t}\left(r_{t}, R\right)$ indicates the illumination of the target

The modified radiometric range equations is then written respectively for totally reflected beam, partially reflected beam and Gaussian beam model

$$
\begin{gathered}
P_{r}=\frac{\tau_{a t m}^{2} \tau_{e} \tau_{r} R_{p}^{2}}{2 R^{2}} \int_{0}^{\infty} \int_{0}^{2 \pi} E_{t}\left(r_{t}, R\right) \cdot \Phi\left(r_{t}, R\right) \cdot r_{t} d r_{t} d \varphi_{t} \\
P_{r}=\frac{\tau_{a t m}^{2} \tau_{e} \tau_{r} A_{t} R_{p}^{2}}{2 \pi \theta^{2} R^{4}} \int_{0}^{\infty 2 \pi} \int_{0}^{2 \pi} E_{t}\left(r_{t}, R\right) \cdot \Phi\left(r_{t}, R\right) \cdot r_{t} d r_{t} d \varphi_{t} \\
P_{r}=\left(\frac{\tau_{e} \tau_{r} R_{p}^{2}}{2}\right)\left[\frac{\exp ^{-2 \gamma(V) R}}{R^{2}} \int_{0}^{\infty} \int_{0}^{\infty 2 \pi} E_{t}\left(r_{t}, R\right) \cdot \Phi\left(r_{t}, R\right)\right. \\
\cdot\left[1-\exp \left(\frac{-2 r_{t}^{2}}{\theta^{2} R^{2}}\right)\right] r_{t} d r_{t} d \varphi_{t}
\end{gathered}
$$

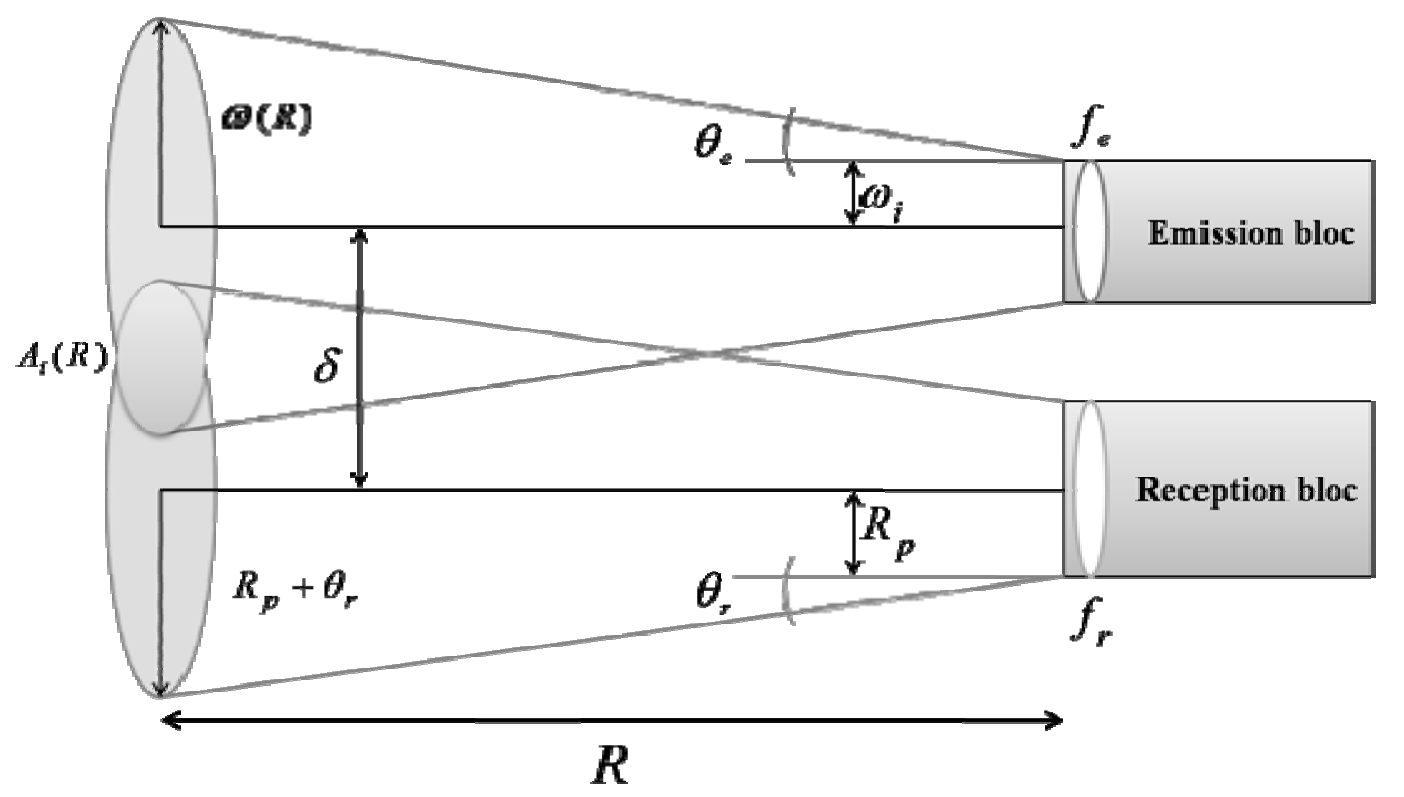

Fig. 5 The intersection area representing the effective fraction of the flux captured by the photodetector. 


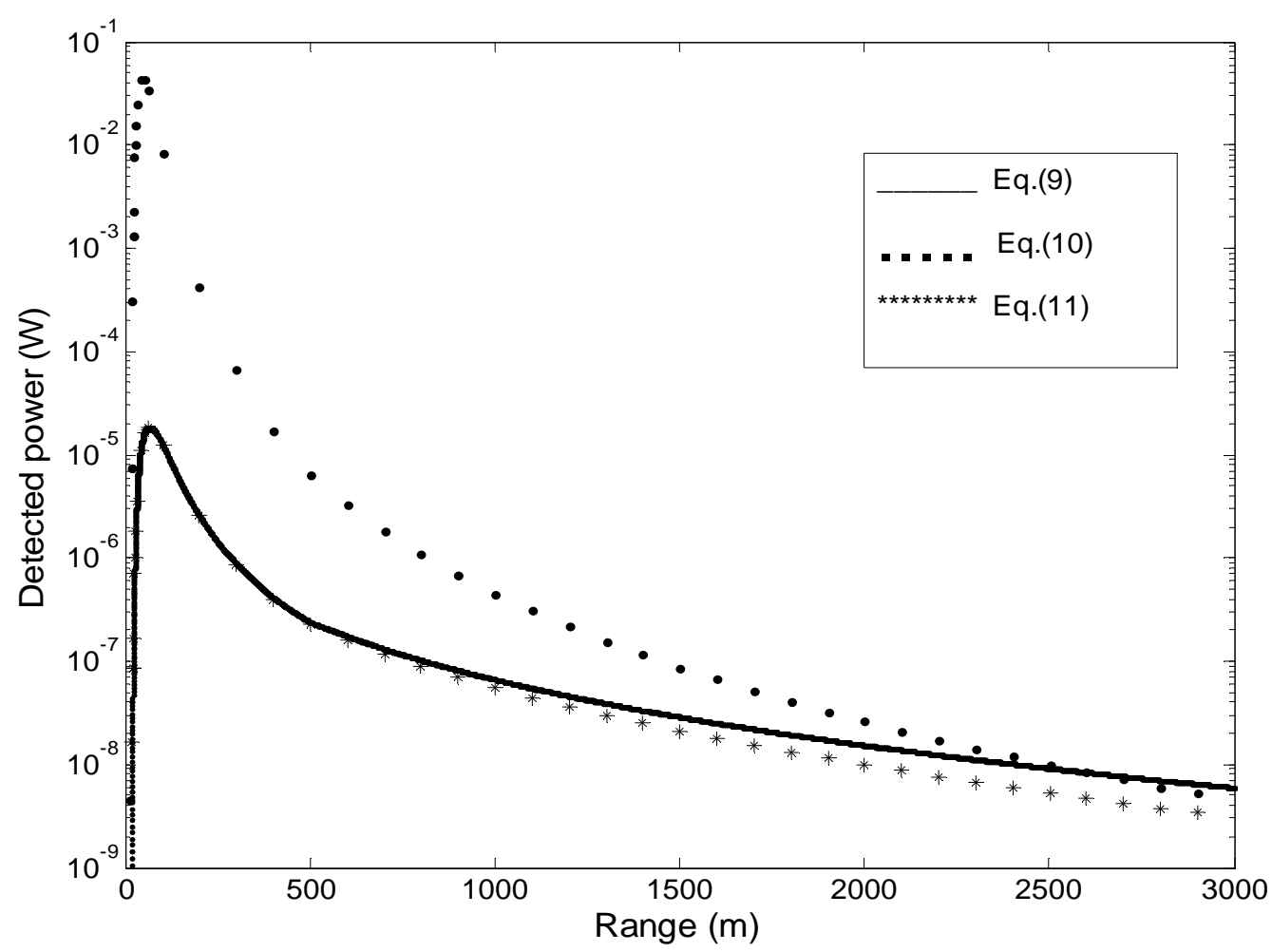

Fig. 6 Plots of Eqs. (9), (10) and (11).

Eq. (11) is a new Gaussian reformulation of radiometric range equation that has never been reported before, taking into account photometric parameters affecting the laser rangefinder detection system.

The numerical simulation results are given below for the three equations with following data:

$$
\begin{gathered}
P_{e}=10^{3} \mathrm{~W}, \quad \rho=0.3, \quad \tau_{e} \times \tau_{r}=0.8 ; \\
V=20,000 \mathrm{~m} ; \\
A_{t}=2.3 \times 2.3 \mathrm{~m}^{2}(\text { NATO standard }) \\
R_{p}=25 \mathrm{~mm}, \theta_{r}=0.5 \mathrm{mrad}, \theta_{e}=0.5 \mathrm{mrad}, \\
\Delta_{\varphi}=0.1 \mathrm{mrad}, d=0.1 \mathrm{~mm}, f=200 \mathrm{~mm}, \\
\delta=50 \mathrm{~mm}, \omega_{i}=0.677 \mathrm{~mm}, D_{\omega_{i}}=100 \mathrm{~mm} .
\end{gathered}
$$

The simulation of the equations of the three radiometric range functions on the basis of photometric parameters shown in Fig. 6 gives precisely the amplitude of the received optical signal as a function of the target distance. The adjustment of the optical and geometrical characteristics of the laser rangefinder reduces the signal span; this is very necessary for not saturate the photodetector, and thus, perform the distance measurements with a linear working of the receiving cell. We note that these equations produce identical results as in the general case reported by $\mathrm{S}$. Kruapech and J. Widjaja [4] from a close distance of 80 $\mathrm{m}$, which justifies the accuracy of the approximation made for a the Gaussian radiometric range equation and confirms more further the validity of this model taking into account, for the first time in this work, in addition to the parameters mentioned before, a new estimates based on geometric and photometric effect that influence practically the range measurement.

The simulation also shows that the classical telemetry equations of general case as well as the Gaussian reformulation, are not valid for a range distance too close to the laser imaging system, which is affected by the intrinsic characteristics of the rangefinder, and the radiometric and photometric influences. All this parameters provide the appropriate imaging laser device for the intended application.

\section{Conclusion}

We have presented in this article a new reformulation of the radiometric Gaussian range 
equation (Eq. (10)) taking into account the photometric parameters affecting the accuracy of targets range measurement and directly influencing the quality of reconstructed images in an imaging laser application. The theoretical study and the simulation presented above, confirms the validity of the proposed photometric Gaussian model and puts more restriction on the short ranges, even in optimal conditions of visibility .

Otherwise, it is necessary to specify the exact range of the maximum sensing distance depending on the visibility not to exceed between the laser imaging system and the scenes (targets) to reconstruct, and the minimum distance below which the signal span affect negatively the quality of detected signal. The new photometric Gaussian range equation reformulation provides more opportunities to work in the case of an active sensing (mobile device) without prior hypothesis about the nature of the targets (totally reflective or partially reflective), which substantially improves the practical conditions for active laser imaging applications.

\section{References}

[1] Schwarz, K. P. and EL-Sheimy, N. 2004. "Mobile Mapping Systems-State of the Art and Future Trends." ISPRS Congress, Istambul, Turkey.

[2] Stitch, M. L. 1972. "Laser Range Finding." In Laser Handbook, edited by Arecchi, F. T. and Schulz-Dubois, E. O., Amsterdam, North -Holland. 1745-804.

[3] Amann, M. C., Bosch, T., Lescure, M., Myllyla, R. and Rioux, M .2001. "Laser Ranging: A Critical Review of Usual Techniques for Distance Measurement.” Opt Eng 40: $10-9$.

[4] Kruapech, S. and Widjaja, J. 2010. "Laser Range Finder Using Gaussian Beam Ranks Equation.” Optics \& Laser Technology 42: 749-54.

[5] Forrester, P. A. and Hulme, K. F. 1981. "Laser Range Finder." Opt. Quant. Elect. 13: 259-93.

[6] Delaye, V. and Labeye, P. 2000. "High Resolution Eye Safe Laser Time of Flight Range Finding." Ph.D. thesis,
The National Polytechnics Institut of Grenoble: 56-57, 65-76.

[7] Moller, K. D .1988. "Radiometry and Photometry." Optics. Mill Valley California, University Science Books. 378-84.

[8] Sabatini, R. and Richardson, A . 2010 . "Airborne Laser Systems Testing and Analysis." NATO Reserch and Technology Organization.

[9] Jelalian, A. V. 1992. "Laser Radar Systems." Artech House.

[10] Minkoff, J. 1992. “ Review of Noise And Random Processes" and "Coherent and Nocoherent Detection and Processing" Signals, Noise \& Active Sensors, Wiley Interscience: $51-5$ and 117-29.

[11] Wolfe, W. L. and Zissis, G. J. 1993. "The Infrared Handbook." IRIA Series in Infrared \& Electro Optics, ERIM.

[12] Hybrid Eye Safe Laser Range Finder Receiver. Model 759, Analog Modules, Inc., 126 Baywood Avenue, Longwood, FL,

USA. http://www.analogmodules.com/admincenter/datasheets/7 59a.pdf.

[13] Hutt, D. L. and et al. 1994. "Estimating Atmospheric Extinction for Eye Safe Laser Rangefinders." Optical Engineering 33 (11): 3762-73.

[14] Meyzonnette, J. L. and Lépine, T. 1999. "Bases de Radiométrie Optique." Cepadues.

[15] Sternberg, H., Caspary, W., Heister, H. and Klemm, J. 2001. "Mobile Capturing On Roads and Railways Utilizing The Kinematic Survey System KiSS.” The 3rd International Symposium on Mobile Mapping Technology, January 3-5, Cairo, Egypt.

[16] Blug, A., Baulig, C., Dambacher, M., Wölfelschneider, H. and Höfler, H. 2007. "Novel Platform For Terresterial 3D Mapping From Fast Vehicles, PIA07-Phtogrammertric Image Analysis." Munich, Germany, September 19-21:vol 36, part 3/W49B. pp 7-13.

[17] Qingwu, H., Zhiyong, C. and Sheng, G. 2007. "Road Facility Investigation Based on MMT." MMT'07, Mobile Mapping Technologies Symposium, Padua-Italy, 28-31May.

[18] Mei, C. and Rives, P. 2007. "Laser-Augmented Omnidirectional Vision for 3D Localisation and Mapping." INRIA Sophia Antipolis, ICARE Project,France.

[19] Dho, S. W. and et al. 1997. "Application of Geometrical form Factor in Differential Absorption Lidar Measurement." Optical Review 4: 521-6. 